\title{
First diabetic retinopathy prevalence study in Portugal: RETINODIAB Study-Evaluation of the screening programme for Lisbon and Tagus Valley region
}

\author{
Marco Dutra Medeiros, ${ }^{1,2,3}$ Edgar Mesquita, ${ }^{4}$ Ana Luísa Papoila, ${ }^{5,6,7}$ Victor Genro, ${ }^{1}$ \\ João Filipe Raposo ${ }^{1,8}$
}

For numbered affiliations see end of article.

Correspondence to Dr Marco Dutra Medeiros, Portuguese Diabetes Association (APDP), Rua Salitre 118, Lisbon 1250-203, Portugal; marcodutramedeiros@gmail. com

Received 4 February 2015 Accepted 10 March 2015

To cite: Dutra Medeiros $M$, Mesquita E, Papoila AL, et al. Br I Ophthalmol Published Online First: [please include Day Month Year] doi:10.1136/

bjophthalmol-2015-306727

\begin{abstract}
Background/aims In Portugal, so far, there is no study or even accurate data on the prevalence of diabetic retinopathy $(D R)$, based on a large representative sample and on a long-term follow-up. The objective of our study was to determine the prevalence of DR based on a national screening community-based programme. Methods A 5-year retrospective analysis of the RETINODIAB screening programme results was implemented in Lisbon and Tagus Valley area between July 2009 and October 2014. We estimated the prevalence of retinopathy for all patients with type 2 diabetes and studied the association between known risk factors and retinopathy emergence at their first screening. Results Throughout this period, from a total of 103102 DR readable screening examinations, 52739 corresponded to patients who attended RETINODIAB screening at entry. Globally, DR was detected in 8584 patients (16.3\%). Of these, 5484 patients (10.4\%) had mild non-proliferative (NP) DR, 1457 patients (2.8\%) had moderate NPDR and 672 (1.3\%) had severe NPDR. Finally, 971 patients (1.8\%) had proliferative DR requiring urgent referral to an ophthalmologist. The presence of any $D R$, non-referable DR or referable DR was strongly associated with increasing duration of diabetes and earlier age at diagnosis.

Conclusions The prevalence rate of DR in our study $(16.3 \%)$ was slightly lower than other published international data. The RETINODIAB network proved to be an effective screening programme as it improved DR screening in Lisbon and Tagus Valley surrounding area.
\end{abstract}

\section{INTRODUCTION}

Diabetic retinopathy (DR) is the leading cause of legal blindness in the working-aged population of industrialised societies. ${ }^{1-3}$ In 2013, 382 million people had diabetes; this number is expected to rise to 592 million by 2035, according to the International Diabetes Federation (IDF). ${ }^{45}$

It is well established that the effectiveness of the laser treatment depends on the accuracy and timely treatment of DR among patients with diabetes mellitus, particularly those with a high risk of DR. ${ }^{6}$ Indeed, DR represents an excellent paradigm for screening as laid out in the principles for screening of human disease described by Wilson and Jungner in $1968 .^{78}$

In order to decrease by about $30 \%$ the new cases of blindness caused by diabetes, the declaration of St. Vincent (1989) called for the implementation of national strategies for screening for DR in a systematic manner. ${ }^{9}$ WHO, IDF and the DirectorateGeneral of Health (DGS) co-organised (1997) the Fourth Meeting in Lisbon for the Implementation of the St. Vincent Declaration on Diabetes Care and Research in Europe, which was attended by delegates from 60 countries. ${ }^{10}$ This conference reinforced once again the need for greater engagement from all signatory countries for the St. Vincent Declaration in order to address diabetes complications, particularly DR. This international challenge was strengthened at the Liverpool meeting in $2005 .{ }^{11}$ Despite all, it is only in the last decade that significant progress has been made in implementing screening programmes to detect and monitor DR.

Portugal currently has a population of 10.6 million, predominantly Caucasian, whose majority (around 8.5 million) is located on the western coast $(\sim 80 \%) .{ }^{12}$ According to the National Observatory for Diabetes, nearly one million Portuguese have diabetes, the equivalent to $13 \%$ of the population between 20 and 79 years. $^{13}$ Of these, about 400000 people are undiagnosed. It is imperative that these people are identified through early diagnosis in order to significantly reduce the incidence of serious complications.

The Portuguese Diabetes Association (APDP) is the world's oldest diabetes association and a senior member of the IDF. From the moment it was founded, early in the 20th century, APDP has been driven by a single overarching objective: to improve the quality of life of people with diabetes. Involved nationally in diabetes advocacy and the provision of education, as well as the delivery of care, APDP has become a key player in the healthcare arena in Portugal.

Following a pilot regional DR screening programme which was launched in 2008, the Diabetic Retinopathy Screening Service for Lisbon and Tagus Valley-RETINODIAB-was commissioned and driven by APDP. This screening programme is supported by the Regional Health Administration of Lisbon and Tagus Valley (ARSLVT) and follows the norms of the DGS, which is a public body of the Ministry of Health. The major aim of the programme was to identify all undiagnosed sightthreatening DR in order to ensure timely onward referral to Lisbon area hospital eye services.

Herein, the authors describe the first Portuguese study regarding the prevalence of DR, as well as focus on the screening programme for DR (20092014) implemented in the area of Lisbon and Tagus Valley. 


\section{METHODS}

\section{The RETINODIAB network}

RETINODIAB (Study Group for Diabetic Retinopathy Screening) is a m-health screening system carried out by APDP, which focuses on clinical aspects of DR screening. Its primary aim is to promote the advancement of knowledge on all aspects of DR through an active cooperation between ophthalmologists and other specialists such as endocrinologists, internists and neurologists. Additionally, APDP has fostered the development of important scientific studies in epidemiology and diabetology in Portugal. ${ }^{14} 15$

\section{Lisbon and Tagus Valley area}

Lisbon and Tagus Valley is one of the five Regions of Portugal (Nomenclature of Territorial Units for Statistics (NUTS) II divisions). It corresponds to $13 \%$ of the Portuguese territory, it has a population of 3.7 million (34.7\% of the total population) and it represents $44 \%$ of the national Gross domestic product (GDP). There are 15 primary care groups (ACES) in this area (figure 1) organised according to the five existing NUTS III (subregions: Greater Lisbon, Setúbal Peninsula, Middle Tagus and Lezíria West Coast).

\section{APDP screening protocol-RETINODIAB}

The RETINODIAB screening programme was held in several primary care health units covered by the APDP protocol. Each screening centre is equipped with a non-mydriatic camera (model CR-2, Canon, Tokyo, Japan). Two $45^{\circ}$ non-stereoscopic retinal digital photographs per eye were taken in a scotopic environment, one centred on the posterior pole and the other on the optic disc. Despite all efforts, in several patients it was impossible to obtain an image with minimum quality. In these specific cases, orthoptists have proceeded to iatrogenic pupil dilation. The remaining possible causes for deficient acquisition of fundus were documented in the clinical report and those patients were referred to a specialist within a maximum period of 3 months. After the capture of images, they are compressed in the Digital Imaging and Communications in Medicine (DICOM) protocol and transmitted through the internet to APDP reading centre. All images were classified according to The International Clinical Diabetic Retinopathy Scale. ${ }^{16}$ As clinically significant macular oedema is not discernible on nonstereoscopic images, maculopathy was defined as the presence of hard exudates or haemorrhages within 1 disc diameter of the fovea. Patients who had undergone panretinal laser treatment

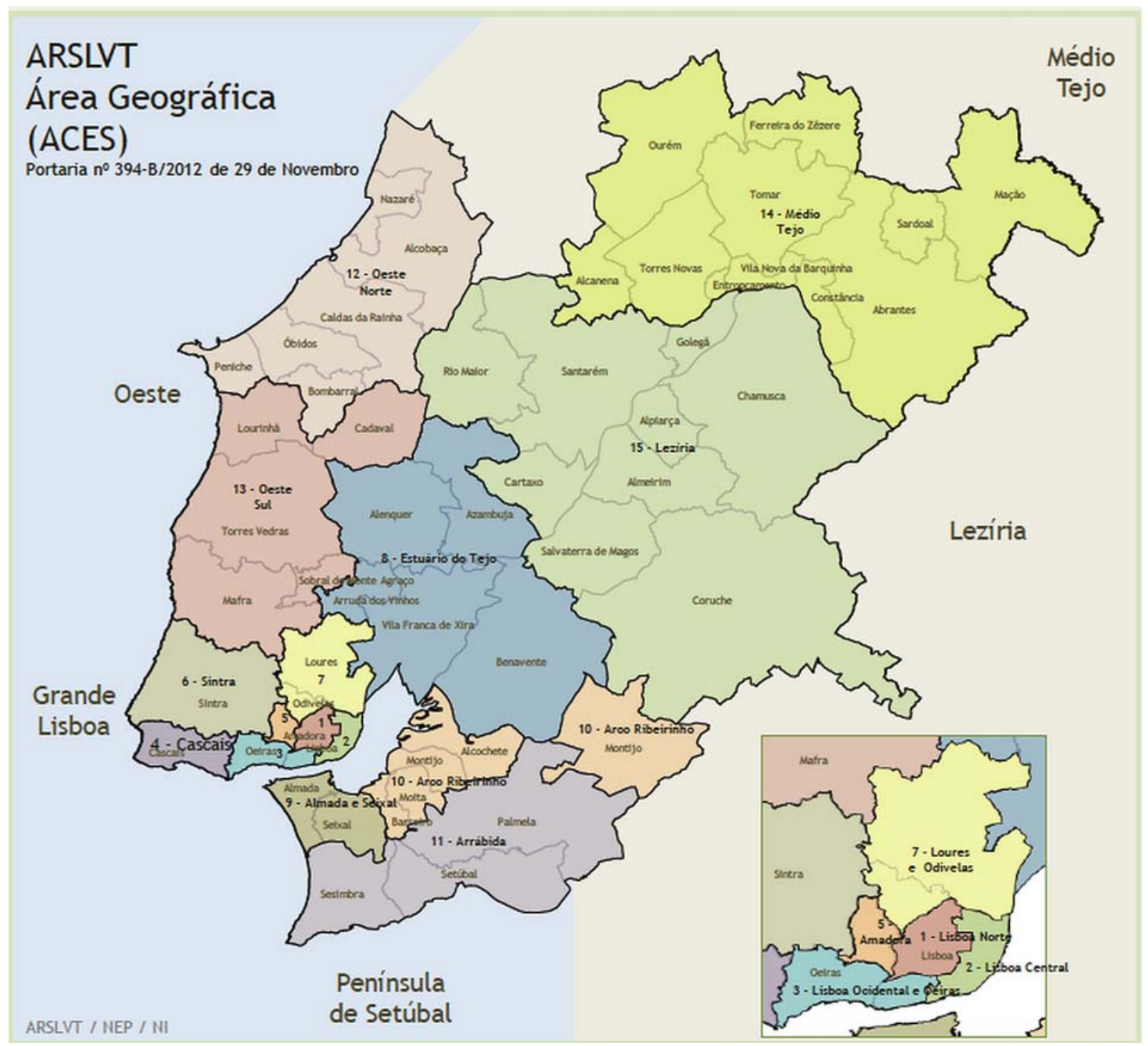

Figure 1 Regional Health Administration of Lisbon and Tagus Valley geographic area. 
were classified as having proliferative retinopathy. Both eyes were assessed for DR and the worse grade from the two eyes was used in the analysis. Retinal images were considered not gradable if retinas of both eyes could not be visualised properly - that is, retinal vessels were not visible within 1 disc diameter of the fovea and fine vessels were not visible across the surface of the optic disc. Where only one eye was gradable, the presence or absence of DR relied on this eye. The reader-automatically generated report displays diagnosis of DR level, diagnosis of non-diabetic ocular disorders and recommendations for follow-up (figure 2).

\section{APDPSoft software}

The APDPSoft is a software developed since 1999, which accompanies the evolution of the services provided by the APDP. Currently, this software supports and monitors several valences, especially in terms of clinical data file, markings management, laboratory parameters, invoicing the health subsystems, integration of numerous diagnosis equipments as well as an effective liaison with the electronic services of the Ministry of Health. It makes the storage of clinical data, fundus photographs and statistics. All stored images were downloaded by certified ophthalmologists at the RETINODIAB Reading Centre comprising three readers.

\section{Statistical methods}

The features of the study participants were described using means (SD) for continuous variables and percentages for categorical variables. The age at diagnosis was categorised into four groups (40-49 years, 50-59 years, 60-69 years and $>70$ years) for all analyses at first screening. The duration of diabetes was discretised into four categories $(<5$ years, 5-9 years, $10-15$ years and $>15$ years). Adjusted ORs and corresponding 95\% CIs were calculated. Furthermore, we defined referable diabetic retinopathy (RDR) to all patients graded as moderate non-proliferative (NP) DR, severe NPDR or proliferative retinopathy DR (with or without maculopathy) or maculopathy with mild retinopathy. This category relates to those who would, according to the guidelines, need referral to the hospital eye service for further clinical evaluation. Univariable and multivariable logistic regression models were used to assess the association of the collected variables with retinopathy status, separately for each subset of diabetes (any DR; RDR; nonreferable diabetic retinopathy (NRDR)). A level of significance of $\alpha=0.05$ was considered. All data were analysed using the Statistical Package for the Social Sciences for Windows V.22.0 (IBM Corp. Released 2013. IBM SPSS Statistics for Windows, V.22.0. Armonk, New York: IBM Corp.).

\section{RESULTS}

Our 5-year retrospective analysis included data for all patients with type 2 diabetes, diagnosed over the age of 40 years, and who attended APDP SP between July 2009 and October 2014.

Throughout this period, from a total of 103102 DR readable screening examinations, 52739 corresponded to patients who attended RETINODIAB screening at entry. The baseline features of study participants are included in table 1 . Patients' mean age is $69.13(\mathrm{SD}=11.13)$ years. Women accounted for $49.6 \%$ $(n=26149)$ of all patients. The mean duration of diabetes was 8.5 years $(\mathrm{SD}=7.89)$.

Overall, not all screening examinations resulted in assessable images. In our present study, retinal photographs of at least one eye could not be graded in 2757 of the 55496 total screening patient examinations performed at entry (4.96\%). This subset of patients was not included in the final group used to calculate several prevalence rates (total number of DR diagnoses performed).

The prevalence of the different categories of DR is shown in table 2, regarding to the total number of assessable images.

Globally, DR was detected in 8584 patients (16.3\%). Of these, 5484 patients (10.4\%) had mild NPDR, 1457 patients $(2.8 \%)$ had moderate NPDR and $672(1.3 \%)$ had severe NPDR. Finally, 971 patients $(1.8 \%)$ had proliferative DR requiring urgent referral to an ophthalmologist. A total of 732 patients $(1.4 \%)$ had maculopathy.

The results of the multivariable logistic regression analysis are shown in table 3 .

Men had increased odds of all severities of DR compared with women. The odds of all grades of DR increased with the duration of diabetes. There was a 2.50-fold, 4.99-fold and 8.20-fold increased odds of any DR associated with a duration

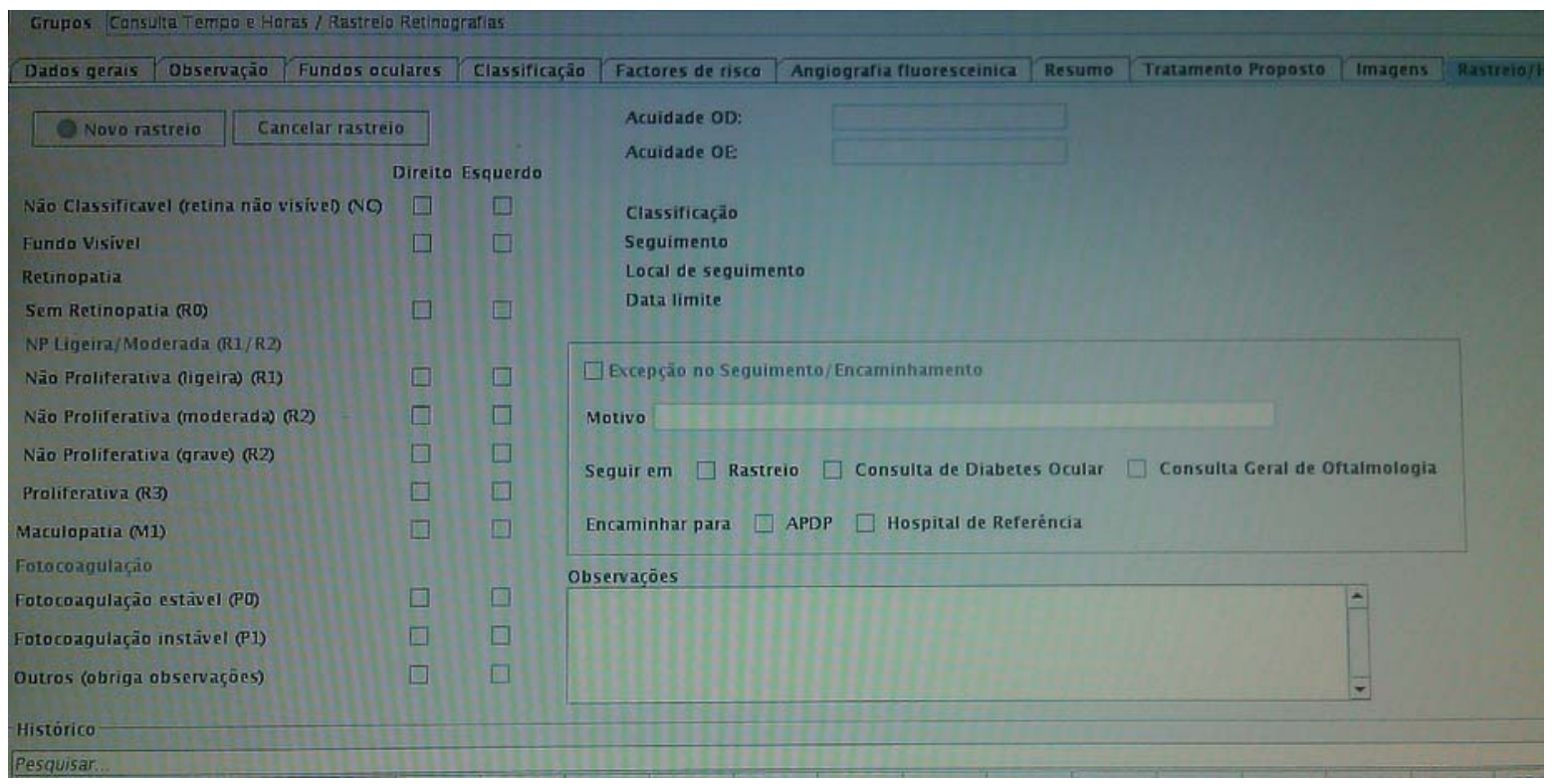

Figure 2 Layout for a reader-automatically generated report before its execution. 
Table 1 Baseline features of study participants at time of first screening event

\begin{tabular}{ll}
\hline Features & Type 2 diabetic patients \\
\hline Total number of patients screened & 55496 \\
Total number of diagnoses done* & 52739 \\
Patients screened with unassessable images & 2757 \\
Gender $n(\%)$ & \\
$\quad$ Male & $26590(50.4)$ \\
$\quad$ Female & $26149(49.6)$ \\
Mean age, years & $69.13(11.13)$ \\
Mean age at diagnosis, years & $60.63(12.15)$ \\
Mean duration of diabetes, years & $8.5(7.89)$ \\
Treatment of diabetes & \\
$\quad$ Oral hypoglycemic agents/diet only & $50581(95.9)$ \\
Insulin & 2157 (4.1) \\
\hline *Total number of examinations with gradable photographs (used to calculate \\
prevalences).
\end{tabular}

of diabetes of $5-9,10-15$ and $\geq 15$ years compared with $<5$ years (reference subgroup) and a 2.38-fold, 4.19-fold and 5.03 -fold increased odds of NRDR in the same subgroups, respectively. For RDR subset of patients, the odds increased by a factor of 2.80 with a known duration of diabetes of 5-9 years and 6.37-fold for a known duration of diabetes of 10-15 years. Finally, for patients with a duration over 15 years the odds increased 12.43-fold compared with the reference subgroup.

Additionally, the ORs of any DR, NRDR and RDR groups were significantly lower with the increasing of age at diagnosis of diabetes. For instance, the odds of any DR increased to 1.12, to 1.48 and to 2.00 in the age ranges $60-69$ years, 50-59 years and $>$ under 50 years, respectively, compared with the reference group ( $>70$ years).

Regarding insulin treatment, all patients with diabetes under insulin therapy had a significant increased odds for all different DR subgroups.

Table 2 The prevalence of diabetic retinopathy (DR) at first screening for all patients successfully screened

\begin{tabular}{lrll}
\hline DR status & Patients & Per cent & 95\% Cl \\
\hline Total & 52739 & $100 \%$ & \\
No DR & 44155 & 83.7 & \\
Any DR & 8584 & $16.3 \%$ & (16 to 16.5) \\
$\quad$ Mild NPDR & 5484 & 10.4 & (10.1 to 10.7) \\
Moderate NPDR & 1457 & $2.8 \%$ & (2.6 to 2.9) \\
Severe NPDR & 672 & $1.3 \%$ & $(1.2$ to 1.4) \\
PDR & 971 & $1.8 \%$ & $(1.7$ to 2.0) \\
Maculopathy & 732 & $1.4 \%$ & $(1.3$ to 1.5) \\
Non-referable DR & & & \\
Mild NPDR without maculopathy & 5258 & $9.99 \%$ & (9.9 to 10.1) \\
Referable DR & 3326 & $6.31 \%$ & (6.2 to 6.4) \\
Mild NPDR with maculopathy & 226 & & \\
Moderate NPDR without maculopathy & 1220 & & \\
Moderate NPDR with maculopathy & 237 & & \\
Severe NPDR without maculopathy & 516 & & \\
Severe NPDR with maculopathy & 156 & & \\
PDR without maculopathy & 858 & & \\
PDR with maculopathy & 113 & & \\
\hline NPDR, non-proliferative diabetic retinopathy; PDR, proliferative diabetic retinopathy. & & & \\
& & & \\
\hline
\end{tabular}

\section{DISCUSSION}

Until now, there have been no studies addressing the prevalence of DR in Portuguese type 2 diabetic population, which include a large sample size and a long-term follow-up.

Diabetes has a high prevalence in Portugal. The PREVADIAB Study ${ }^{14}$ which was carried out by APDP found a diabetes prevalence of $11.7 \%$, with a significant difference between men $(14.2 \%)$ and women (9.5\%). While 6.6\% (approximately 508 000 people) had previously been diagnosed with diabetes, 5.1\% (around 393000 persons) were undiagnosed. On the other hand, the prevalence of 'pre-diabetes' (impaired fasting glucose, impaired glucose tolerance or both) in the population was $23.3 \%$. In our study, we reported a prevalence of any DR, NRDR and RDR in patients with type 2 diabetes of $16.3 \%$, $10.4 \%$ and $5.9 \%$, respectively.

The teleophthalmology network constitutes an efficient means to overcome the lack of ophthalmologists. ${ }^{17}$ In accordance with the aforementioned, Portugal may have about one million people with diabetes, of whom 700000 diagnosed and on medical treatment and who should be consulted annually according to the criteria stated above. According to the Portuguese Ophthalmology Society (SPO), each of the 930 Portuguese ophthalmologists (2012 data) would have to observe about 753/each year, an infeasible number in terms of logistics specialty requirements. Moreover, screening centres or mobile units using non-mydriatic cameras should be allocated in areas with a high rate of poverty and a low number of ophthalmologists, like the West Region of Portugal, which is covered by RETINODIAB programme. Additionally, the centralisation of the network around a central reading headquarters provides a quality control and uniformity between graders.

The epidemiological studies addressing DR prevalence in type 2 diabetes have varied worldwide, at least partly due to different ethnic populations and different sample sizes. ${ }^{18} 19$ Nevertheless, the comparison of the DR prevalence rates between published studies is difficult due to the lack of uniformity regarding the different grading protocols employed. In France, Massin et al carried out several epidemiological studies addressing DR screening in Paris and the surrounding area. ${ }^{20}$ They documented a prevalence of any DR around $24 \%$. Several UK screening programmes evaluated the prevalence of DR for type 2 diabetes. The Scottish programme ascertained the DR prevalence in 47090 newly diagnosed patients with type 2 diabetes and reported at $19.3 \%$ for any DR and $1.9 \%$ for RDR. ${ }^{21}$ Furthermore, Thomas et $a l^{22}$ undertook a cross-sectional analysis of 86390 patients with type 2 diabetes in Wales. They documented a prevalence of any DR and sight-threatening DR of $30.3 \%$ and $2.9 \%$, respectively. Similarly, the presence of DR, non-sight-threatening and sight-threatening, was strongly associated with either variables: increasing duration of diabetes and earlier age at diagnosis. In Iceland, the prevalence of DR was higher in type 2 diabetes, estimated at $41.0 \% .^{23}$ The prevalence rate of DR in our study (16.3\%) was slightly lower than other published data. Indeed, all patients previously diagnosed with DR had been already previously forwarded, and only patients whose retinal status was unknown were included in this study.

Regarding logistic regression analysis, the duration of diabetes was a significant risk factor for the presence of any DR, NRDR and referable diabetic retinopahy (RDR) in subjects with type 2 diabetes. Multivariable adjusted ORs were much higher in all subgroups, in the longer time frame (over 15 years), compared with other shorter periods of time. The strong association with disease duration demonstrates the importance of early detection and enrolling to a screening programme. Moreover, a later age 
Table 3 Multivariable logistic regression analysis for the association between age at diagnosis, gender, duration of diabetes and diabetes treatment with the presence of any diabetic retinopathy (DR), non-referable diabetic retinopathy (NRDR) and referable diabetic retinopathy (RDR)

\begin{tabular}{|c|c|c|c|}
\hline & $\begin{array}{l}\text { Any DR } \\
\text { OR }(95 \% \mathrm{Cl})\end{array}$ & $\begin{array}{l}\text { NRDR } \\
\text { OR }(95 \% \mathrm{Cl})\end{array}$ & $\begin{array}{l}\text { RDR } \\
\text { OR }(95 \% \mathrm{Cl})\end{array}$ \\
\hline \multicolumn{4}{|l|}{ Age at diagnosis of diabetes } \\
\hline$\geq 70$ years $(n=12678)$ & 1.00 & 1.00 & 1.00 \\
\hline $60-69$ years $(n=16443)$ & $1.12(1.02 \text { to } 1.21)^{*}$ & $1.06(0.97$ to 1.16$)$ & $1.34(1.15 \text { to } 1.56)^{*}$ \\
\hline $50-59$ years $(n=14569)$ & $1.48(1.36 \text { to } 1.61)^{*}$ & $1.34(1.22 \text { to } 1.47)^{*}$ & $1.89(1.62 \text { to } 2.19)^{*}$ \\
\hline$<50$ years $(n=9049)$ & $2.00(1.83 \text { to } 2.19)^{*}$ & $1.61(1.45 \text { to } 1.78)^{*}$ & $2.73(2.34 \text { to } 3.19)^{*}$ \\
\hline \multicolumn{4}{|l|}{ Gender } \\
\hline Female $(n=26$ 149) & 1.00 & 1.00 & 1.00 \\
\hline Male $(n=26590)$ & $1.23(1.17 \text { to } 1.30)^{*}$ & $1.16(1.10 \text { to } 1.23)^{*}$ & $1.27(1.18 \text { to } 1.37)^{*}$ \\
\hline \multicolumn{4}{|l|}{ Duration of diabetes } \\
\hline$<5$ years $(n=20322)$ & 1.00 & 1.00 & 1.00 \\
\hline $5-9$ years $(n=12998)$ & $2.50(2.30 \text { to } 2.72)^{*}$ & $2.38(2.17 \text { to } 2.62)^{*}$ & $2.80(2.38 \text { to } 3.29)^{*}$ \\
\hline $10-15$ years $(n=12103)$ & $4.99(4.61 \text { to } 5.40)^{*}$ & $4.19(3.84 \text { to } 4.58)^{*}$ & $6.37(5.50 \text { to } 7.37)^{*}$ \\
\hline$>15$ years $(n=7316)$ & $8.20(7.52 \text { to } 8.94)^{*}$ & $5.03(4.56 \text { to } 5.55)^{*}$ & $12.43(10.70 \text { to } 14.45)^{*}$ \\
\hline \multicolumn{4}{|l|}{ Insulin treatment } \\
\hline No $(n=50581)$ & 1.00 & 1.00 & 1.00 \\
\hline Yes $(n=2157)$ & $3.22(2.93 \text { to } 3.54)^{*}$ & $2.10(1.89 \text { to } 2.33)^{*}$ & 2.90 (2.59 to 3.25$)$ \\
\hline
\end{tabular}

of diagnosis has a protective effect regarding all grades of DR. Indeed, all patients who were diagnosed over the age of 70 years have twofold lower risk of developing any DR compared with all patients with diabetes diagnosed under 50 years old. This is in accordance with several studies that advise a variable schedule distribution of screening intervals according the individual patients' risk. In fact, increasing the length of the screening intervals for lower risk cases would involve less screening episodes, with resulting benefits in terms of health costs. $^{24}$

The main purpose of RETINODIAB implementation was to improve DR screening in Lisbon and Tagus Valley surrounding area in order to efficiently perform, within an acceptable time frame, all eye examinations according to the guidelines of the DGS. ${ }^{25}$

This study provides the first estimate of the prevalence of DR for subjects over the age of 40 years and not receiving ophthalmological assistance in Portugal. In line, to the best of our knowledge, this study represents the second largest reported international community-based DR screening network. We will continue to follow these patients to better define all clinical and epidemiological data regarding this diabetic population.

\section{Author affiliations}

${ }^{1}$ Portuguese Diabetes Association APDP, Lisbon, Portugal

${ }^{2}$ Department of Ophthalmology, Central Lisbon Hospital Center, Lisbon, Portugal

${ }^{3}$ NOVA Medical School, Universidade NOVA de Lisboa, Lisbon, Portugal

${ }^{4}$ University of Minho, Braga, Portugal

${ }^{5}$ CEAUL, Lisbon, Portugal

${ }^{6}$ Statistics and Informatics Department, NOVA Medical School, Universidade NOVA de Lisboa, Lisbon, Portugal

${ }^{7}$ Epidemiology and Statistics Unit, Research Centre, Central Lisbon Hospital Center, Lisbon, Portugal

${ }^{8}$ Department of Public Health/CEDOC, NOVA Medical School, Universidade NOVA de Lisboa, Lisbon, Portugal

Acknowledgements The authors would like to thank all the people involved in the RETINODIAB network, namely computer engineers, administratives, orthoptists and graders.

Contributors All authors have contributed to the planning, conduct and reporting of the work described in the article.

Funding The APDP screening network was supported by a grant from the Portuguese Ministry of Health.

\section{Competing interests None.}

Ethics approval APDP ethics committee, as well as from Directorate-General of Health (DGS), , on behalf of the Ministry of Health.

Provenance and peer review Not commissioned; externally peer reviewed.

\section{REFERENCES}

1 Mokdad AH, Ford ES, Bowman BA, et al. Diabetes trends in the U.S.: 1990-98. Diabetes Care 2000;23:1278-83.

2 MacKinnon JR, Forrester JV. Diabetic retinopathy. In: Wass JAH, Shalet SM, Eds. Oxford textbook of endocrinology and diabetes. Oxford, UK: Oxford University Press, 2002:1764-78.

3 Franck RN. Diabetic retinopathy. New Eng J Med 2004;350:48-58.

4 Guariguata L, Whiting DR, Hambleton I, et al. Global estimates of diabetes prevalence for 2013 and projections for 2035. Diabetes Res Clin Pract 2014;103:137-49.

5 International Diabetes Federation. IDF Diabetes Atlas. 6th edn. Brussels, Belgium: International Diabetes Federation, 2013. http://www.idf.org/diabetesatlas (accessed 21 Nov 2014).

6 Diabetic Retinopathy Study Research Group. Photocoagulation treatment of proliferative diabetic retinopathy: clinical application of Diabetic Retinopathy Study (DRS) findings report number 8. Ophtalmology 1981;88:583-600.

7 Cuckle HS, Wald NJ, Lindenbaum RH. Maternal serum alpha-fetoprotein measurement: a screening test for Down syndrome. Lancet 1984;1:926-9.

8 Wilson JMG, Jungner G. Principles and practice of screening for disease. WHO Chronicle. Geneva: World Health Organization, 1968. 22(11): 473. Public Health Papers, \#34

9 World Health Organization/International Foundation Europe. Diabetes care and research in Europe: the Saint Vincent declaration. Diabetes Med 1990;7:360.

10 Fourth Meeting for the Implementation of the St.Vincent Declaration. Diabetes Care and Research in Europe. Improvement of Diabetes Care. Lisbon, Portugal, 26 February-1 March 1997.

11 Screening for diabetic retinopathy in Europe: 15 years after the Saint Vincent Declaration. Proceedings of the Liverpool Declaration, 17-18 November 2005, Liverpool, UK. http://www.drscreening2005.org.uk (accessed 21 Nov 2014).

12 Census_Final results: Portugal-2011." Statistics Portugal. 20 November 2012. http://www.ine.pt (accessed 25 Nov 2014).

13 Diabetes: Factos e Números 2013- Relatório Anual do Observatório Nacional da Diabetes; Novembro/2013. https://www.dgs.pt/documentos-e-publicacoes/ diabetes-factos-e-numeros-2013.aspx (accessed 6 Dec 2014).

14 Gardete-Correia L, Boavida JM, Raposo JF, et al. First diabetes prevalence study in Portugal: PREVADIAB study. Diabet Med 2010;27:879-81.

15 Pinto-Figueiredo L, Moita J, Genro V, et al. Diabetic retinopathy in a population of 1,302 insulin dependent diabetics (IDDM) diagnosed before 30 years of age. Int Ophthalmol 1992;16:429-37. 


\section{Clinical science}

16 Wilkinson CP, Ferris FL III, Klein RE, et al. Global Diabetic Retinopathy Project Group. Proposed international clinical diabetic retinopathy and diabetic macular edema disease severity scales. Ophthalmology 2003;110:1677-82.

17 Massin P, Erginay A, bem Mehidi A, et al. Evaluation of a new non-mydriatic digital camera for detection of diabetic retinopathy. Diabet Med 2003;20:635-41.

18 Williams R, Airey $\mathrm{M}, \mathrm{Baxter} \mathrm{H}$, et al. Epidemiology of diabetic retinopathy and macular edema: a systematic review. Eye (Lond) 2004;18:963-83.

19 Sivaprasad S, Gupta B, Crosby-Nwaobi R, et al. Prevalence of diabetic retinopathy in various ethnic groups: a worldwide perspective. Surv Ophthalmol 2012;57:347-70

20 Schulze-Döbold C, Erginay A, Robert N, et al. Ophdiat( (®): five-year experience of a telemedical screening programme for diabetic retinopathy in Paris and the surrounding area. Diabetes Metab 2012;38:450-7.
21 Looker HC, Nyangoma SO, Cromie D, et al. Scottish Diabetic Retinopathy Screening Collaborative; Scottish Diabetes Research Network Epidemiology Group. Diabetic retinopathy at diagnosis of type 2 diabetes in Scotland. Diabetologia 2012;55:2335-42.

22 Thomas RL, Dunstan FD, Luzio SD, et al. Prevalence of diabetic retinopathy within a national diabetic retinopathy screening service. Br J Ophthalmol 2015;99:64-8.

23 Kristinsson JK, Stefansson $E$, Jonasson $F$, et al. Screening for eye disease in type 2 diabetes mellitus. Acta Ophthalmol Scand 1994;72:341-6.

24 Aspelund T, Thornórisdóttir O, Olafsdottir E, et al. Individual risk assessment and information technology to optimise screening frequency for diabetic retinopathy. Diabetologia 2011;54:2525-32

25 Norma da DGS nº 006/2011 de 27/01/2011 (Diagnóstico Sistemático e Tratamento da Retinopatia Diabética. http://www.dgs.pt/ms/7/default.aspx?pl=\&id=5519\& acess $=0$ (accessed 1 Dec 2014). 


\section{First diabetic retinopathy prevalence study in Portugal: RETINODIAB Study --Evaluation of the screening programme for Lisbon and Tagus Valley region}

Marco Dutra Medeiros, Edgar Mesquita, Ana Luísa Papoila, Victor Genro and João Filipe Raposo

Br J Ophthalmol published online April 2, 2015

Updated information and services can be found at:

http://bjo.bmj.com/content/early/2015/04/02/bjophthalmol-2015-30672

7

These include:

References This article cites 17 articles, 2 of which you can access for free at: http://bjo.bmj.com/content/early/2015/04/02/bjophthalmol-2015-30672 7\#BIBL

Email alerting Receive free email alerts when new articles cite this article. Sign up in the service box at the top right corner of the online article.

Topic Articles on similar topics can be found in the following collections

Collections

Epidemiology (946)

Retina (1463)

\section{Notes}

To request permissions go to:

http://group.bmj.com/group/rights-licensing/permissions

To order reprints go to:

http://journals.bmj.com/cgi/reprintform

To subscribe to BMJ go to:

http://group.bmj.com/subscribe/ 